\title{
A Framework of E-Health Systems Adoption and Telemedicine Readiness in Developing Countries
}

\author{
By Ali Abdullrahim and Rebecca De Coster \\ College of Engineering, Design and Physical Sciences \\ Brunel University London, UK
}

\begin{abstract}
Fundamental transformation of the healthcare sector through informatics is achievable, from technical prospective. However, organisational issues surrounding technology implementation in healthcare setting have not yet received adequate research attentions in developing countries. The aim of this study is to develop a conceptual framework model to determine Telemedicine Readiness (TR) at an organisational level in Libyan healthcare establishments. In addition, Telemedicine Adoption and Outcome Expectations were also examined as further model outcomes. A questionnaire based survey was designed and developed to include 7 items concerning various conceptual subscales. The paper reports the responses of 161 participants targeting mainly Libyan physicians and healthcare staff who are studying for further education in the UK and who have been healthcare providers in Libya. The results show a strong correlation of TR with Human Resources (HR) capability ( $\mathrm{r}=$ $0.535, \mathrm{P}<0.001)$ and with IT Infrastructure $(\mathrm{r}=0.44, \mathrm{P}<0.001)$. The overall Cronbach Alpha coefficient $=0.835$. The factor analysis (validity) illustrated five factors with positive loading $>0.5$. Further, user acceptance, IT infrastructure and HR capability have significant influences on TR (r2 $=0.472, \mathrm{P}<0.001)$. From both reliability test and factor analysis, the framework is considered a valid model to assess $\mathrm{TM}$ readiness.
\end{abstract}

\section{Keywords}

Telemedicine, Chronic Conditions, Value Provision, TM Adoption.

\section{INTRODUCTION}

Information and communications technologies (ICT) are enabling technologies for the healthcare systems and Telemedicine (TM) is one of a number of ICT applications. Telemedicine was defined by reference [1] as "the use of ICT to provide and support health care services when distance separates the participants". Healthcare organizations certainly have good reason to consider TM and e-health systems in determining the best ways to provide high quality healthcare under continuous pressures to reduce costs [2], [3]. Such technology will have implications for improving healthcare access, especially to people in the rural areas and patients with chronic disease, and also improving or reforming the healthcare systems in developing countries. TM can enhance organization's immediate area of operations in healthcare industry where associated with service design. Up to date there is little information in the literature regarding the use of TM in Libya and this suggests the 
need to assess the readiness to adopt and eventually implement and sustain TM technologies and e-health systems. Additionally, TM solutions, such as electronic medical records, are not yet applied in Libya's health establishments. These would enhance coordination of clinicians, reduce inefficiency, increase effectiveness and overall quality, and improve safety. The current study is to inspect the readiness at an organisational level in Libya to adopt and utilise TM technologies in order to provide healthcare to service users for monitoring health chronic conditions such as diabetes, high blood pressure and also for patients with strokes and paralysis, regardless of where they are.

\section{CONCEPTUAL BACKGROUND}

Reference [7] explained that "the healthcare stakeholders have often conflicting goals, including access to services, profitability, high quality, cost containment, safety, convenience, patient-centeredness, and satisfaction. Achieving high value for patients must become the overarching goal of healthcare delivery. Value should always be defined around the patients, and in a well- functioning health care system, the creation of value for patients should determine the rewards for all other actors in the system". The underpinning theory for the current research is value provision to service users where value has been defined by [4] as "the (beneficiaries) overall assessment of the utility of a product based on perceptions of what is received and what is given". The use of TM in support to create value in healthcare system and propose it to deliver services for patients with chronic conditions by monitoring them from distance is the core centre of this study. Based on the existing literature, a conceptual framework was designed and developed shown in Fig. 1, where Telemedicine users' acceptance and Telemedicine Service Access are an "inputs" to the associated organisational capabilities including operations, IT infrastructure, human resources and learning capabilities in which will they have a direct impact on the organisation readiness and adoption of TM [8]-[13].

\section{MATERIAL AND METHODS}

A questionnaire was designed based on the conceptual framework's core constructs and this questionnaire was used to collect the quantitative data using a five points Likert scale and after analysing it, interviews will be conducted with policy makers as being the qualitative data to capture their views and validate the results in order to optimise the conceptual framework. The use of mixed method technique is largely used in the health and IT research field and by combining the two methods; we can obtain a much richer understanding [14]. Ethical approval was obtained to use the students' database of the Libyan Embassy, Cultural Affairs Department. The field research was conducted in the UK distributing a questionnaire through a data base of 620 in population targeting mainly Libyan healthcare providers who are studying for further education in the UK and who have practiced in Libya. The total number of participants dropped to 520 after excluding under-graduate students group and the response percentage was $31 \%$. 


\section{STATISTICAL ANALYSIS}

The survey data of healthcare professionals was analysed using the IBM SPSS software V.20. The data was normally distributed. The relationships among the variables were assessed by Pearson's coefficient and then a linear regression was performed to assess the strength of the relationship between variables. The internal consistency/ reliability were checked by Cronbach's alpha coefficient. The exploratory factor analysis was performed to test the construct validity of the questionnaire. The calculated $\mathrm{P}$ value $<0.05$ was considered statistically significant.

\section{RESULTS}

Fig. 2 shows that a total of participants were consisting of 84 females (52.2\%) and 77 males (47.8\%). Their age range was $22-34$ years old (50.2\%), 35-44 years old (36\%) and $45-54$ years old (10.6\%).

\section{A. Demographic variables and model's output}

The independent t-test between the mean of health provider staff gender and the TR was not statistically significant; $\mathrm{P}=0.87$. ANOVA test between different age range was statistically significant; $\mathrm{P}=0.046$. The finding suggests that the TM outcomes did not influence by gender. However, the age range 35-44 was more optimistic regarding TR comparing to the group of age range 22-34. (See Fig. 3)

Years of experience's groups in this research (1-4, 5-10, 10-20, and 20+) behave differently in terms of the model's output. Fig. 4, shows that the mean difference in TM Outcome Expectation was relevantly high and was statistically significant; $\mathrm{P}=$ 0.005 between groups on TM outcome expectation, and $\mathrm{P}=0.044$ between groups on TR. Multiple comparison data shows that the 5-10 years, and 10-20 years groups significantly had positive opinion in term of TR $(\mathrm{P}=0.010, \mathrm{P}=0.033)$ comparing with the less experienced group (1-4 years).

The different sizes of healthcare providers groups have impact on the model's outcome in this research; P value was 0.006 . The small-sized health provider's group showed their support to the TR than medium-sized health provider's group as shown in Fig. 5.

\section{B. Reliability and Construct Validity of Survey}

From table I, the results of Pearson's correlations demonstrated that the strongest relationships are between TR and the following: HR Management $(\mathrm{r}=0.535$ and $\mathrm{P}<$ 0.001), IT Infrastructure $(r=0.44$ and $P<0.001)$, Service Users Acceptance $(r=0.33$ and $\mathrm{P}<0.001)$, HP User Acceptance $(\mathrm{r}=0.321$ and $\mathrm{P}<0.001)$. TM Access $(\mathrm{r}=$ 0.294), Learning Capabilities $(r=0.28)$ and Operational Capabilities $(r=0.277)$ have low relationships with TR. 
Over all questionnaire reliability shows a value of 0.835 and the values of alpha must not be less than 0.70 . That is why the scale of 38 questions was considered as reliable and satisfactory in terms of internal consistency of the conceptual construction of 7 factors model with its outcomes. The Cronbach's alpha for every single variable equals to $70.4 \%, 78.1 \%, 87.7 \%, 77.7 \%, 82.5 \%, 86.6 \%$ and $88.4 \%$ (see table I).

The highest factor loadings produced after Principal Components Analysis (PCA) were questions related to HP user acceptance, HR management capability, IT Infrastructure, HP learning capability and TM access. These mainly load on the first five components with eigenvalue of 9.164, 4.097, 2.761, 2.45 and 2.211 respectively, which explains, following Varimax rotation, 54.431\% of variance of the total loading dispersion. Nevertheless to mention that all the above items appear to have the Pearson's correlation coefficient from medium to strong with no issue in reliability results.

\section{DISCUSSION}

In terms of the model's outputs, years of clinical experience's groups behaved differently where the 5-10 years, and 10-20 years groups had positive opinion in term of TR comparing with the less experience group (1-4 years). It shows that the more clinical experienced participants think their healthcare organisations are ready to adopt the telemedicine technologies as that might help them on their day to day clinical practices whereas the less experienced participants think otherwise. The finding might contribute to the telemedicine technology to be adopted and utilised for the service users with recommendations to HP managers to educate healthcare staff about TM technologies.

The small-sized healthcare provider's group showed their support to the TR than medium-sized health provider's group. The result contradicts a meta-analysis study [15] on relationship between organizational size and IT innovation adoption where they found that the larger the organization the more likely to be ready to adopt innovative due to their finical capacity and IT infrastructure advantages. However, this study is made on a developing country where the type of small healthcare providers are mostly from the private sector in which the financial resources can be more accessible to spend on new ways to provide a competitive and better quality health care. Whereas the larger healthcare provider tend to be from the public sector where bureaucracy and restrict access to financial resources might affect the readiness of the organisation to adopt telemedicine. 


\section{CONCLUSION}

A conceptual framework was developed with the factors contributing to TR and ehealth systems adoption at an organisational level to adopt and utilise telemedicine technologies in order to provide healthcare to service users in developing countries.

Research integrity depends on the accuracy of the measures used. The results of the validity testing on the survey questionnaire in this study indicated that it is an accurate measure of TR. The processes used to validate the survey questionnaire were thorough and suitable. Additionally, demographic variables and their relationships and influences on TR in Libya were also examined.

\section{ACKNOWLEDGMENT}

We acknowledge the respondents who gave their time to participate in this study. We also acknowledge Libyan Embassy in London for granting us permission to carry out the field research.

\section{REFERENCES}

[1] M. J. Field, E. C. Applications, M. Isbn, T. Pdf, N. A. Press, N. A. Press, N. Academy, N. Academy, and N. A. Press, Telemedicine. 1996.

[2] M. Mackert, P. Whitten, and E. Krol, "Planning Successful Telemedicine and E-Health Systems,” pp. 540-553, 2010.

[3] P. Whitten, B. Holtz, and L. Nguyen, "Keys to a successful and sustainable telemedicine program.,” Int. J. Qual. Stud. Health Well-being, vol. 26, no. 2, pp. 2116, 2010.

[4] V. A. Zeithaml, "Consumer Perceptions Of Price, Quality, And Value : A Means-,” J. Mark., no. 52, p. 21, 1988.

[5] S. L. Vargo and R. F. Lusch, "Evolving to a New Dominant Logic for Marketing,” J. Mark., vol. 68, no. 1, pp. 1-17, 2004.

[6] A. F. Payne, K. Storbacka, and P. Frow, "Managing the co-creation of value," J. Acad. Mark. Sci., vol. 36, no. 1, pp. 83-96, 2008.

[7] M. E. Porter, “New engla nd journal,” Perspective, vol. 363, no. 1, pp. 1-3, 2010.

[8] M. Brear, “Organizational Factors in Health Informatics,” Handb. Res. Informatics Healthc. Biomed. U6 - pp. 410-418, 2006. 
[9] K. Cresswell and A. Sheikh, "Organizational issues in the implementation and adoption of health information technology innovations: An interpretative review," Int. J. Med. Inform., vol. 82, no. 5, pp. e73-e86, 2013.

[10] D. Damigou, F. Kalogirou, and G. Zarras, "Use of Telemedicine Systems and Devices for Patient Monitoring,” pp. 221-223.

[11] D. D. Krause, K. R. Butler, and W. L. May, “Associations between factors affecting access to care and health-related quality of life: Results of a statewide HIV/AIDS cross-sectional study,” AIDS Care, vol. 25, no. 1, pp. 1-8, 2012.

[12] D. J. Doyle, "Ergonomic User Interface Design in Computerized Medical Equipment,” pp. 390-392.

[13] D. J. Doyle, “Safety Issues in Computerized Medical Equipment,” pp. 396398, 2006.

[14] L. Condelli, "Real World Research : Combining Qualitative and Quantitative Research for Adult ESL 1,” pp. 1-34, 2004.

[15] M. A. Hameed and S. Counsell, "Establishing Relationships Between Innovation Characteristics and It Innovation Adoption in Organisations: a MetaAnalysis Approach,” Int. J. Innov. Manag., vol. 18, no. 01, p. 1450007, 2014. 


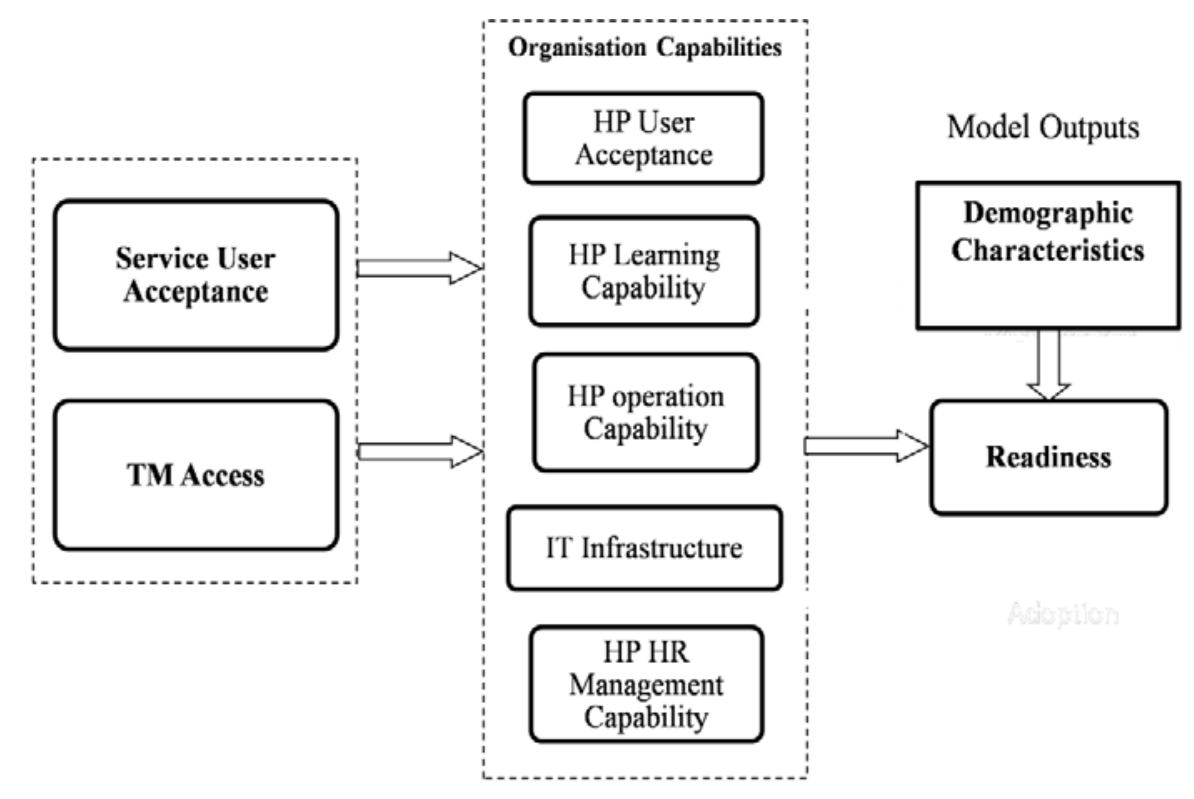

TM: telemedicine; HP: health provider; HR: human resources.

Figure 1. Conceptual framework
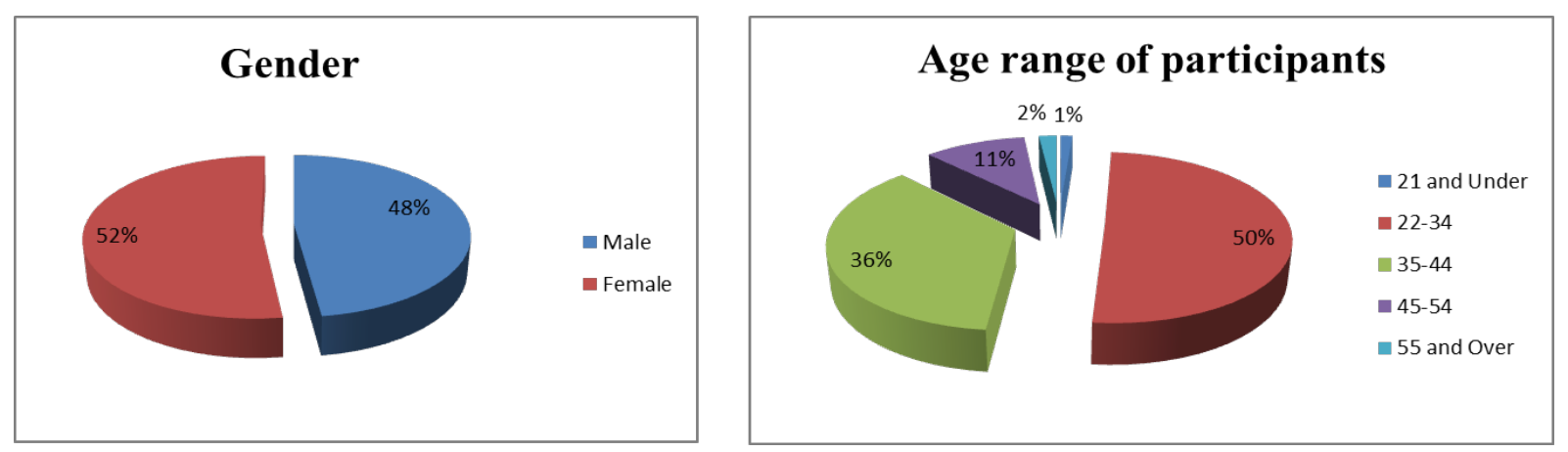

Figure 2. Gender and age range of participants 


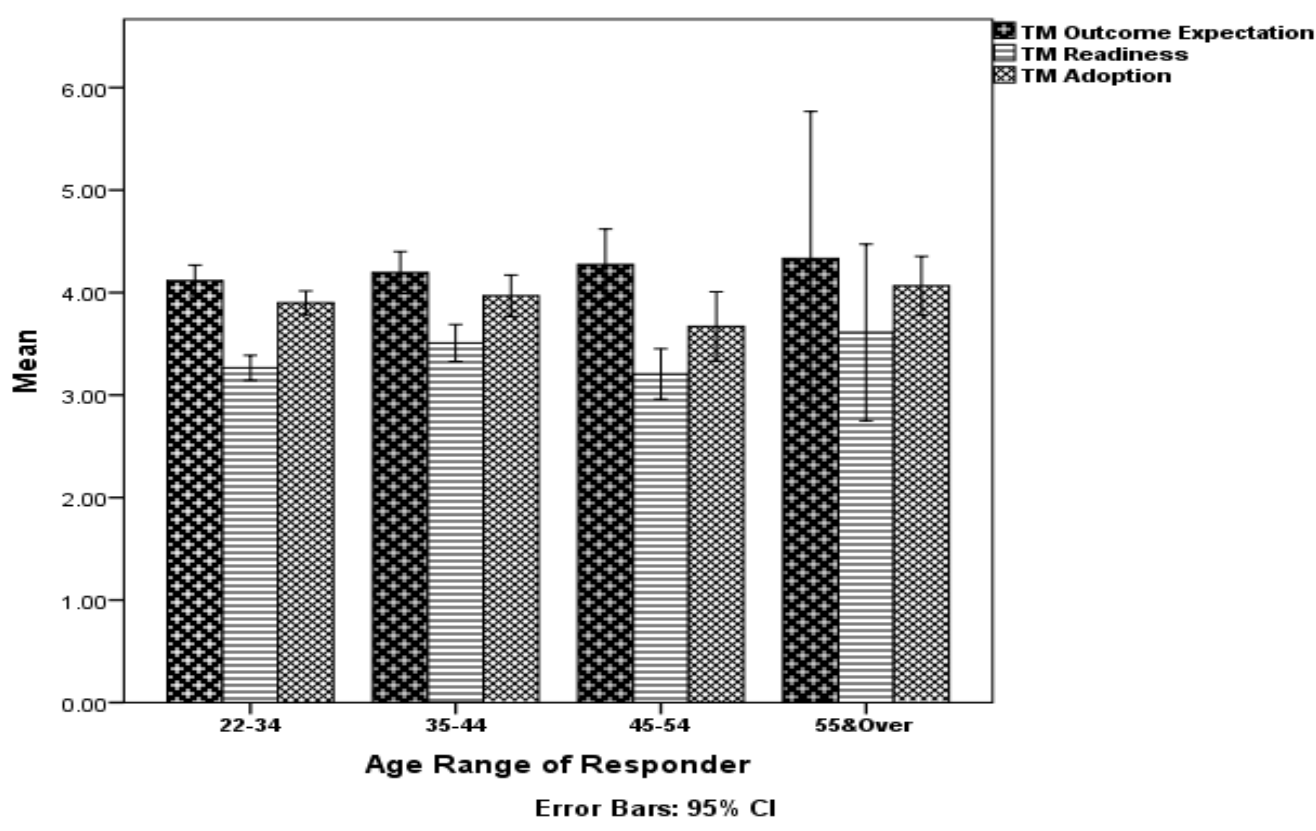

Figure 3. Age range (22-34 yrs old, 35-44 yrs old, 45-54 yrs old and $\geq 55$ yrs old) and telemedicine and readiness. $\mathrm{P}=0.046$

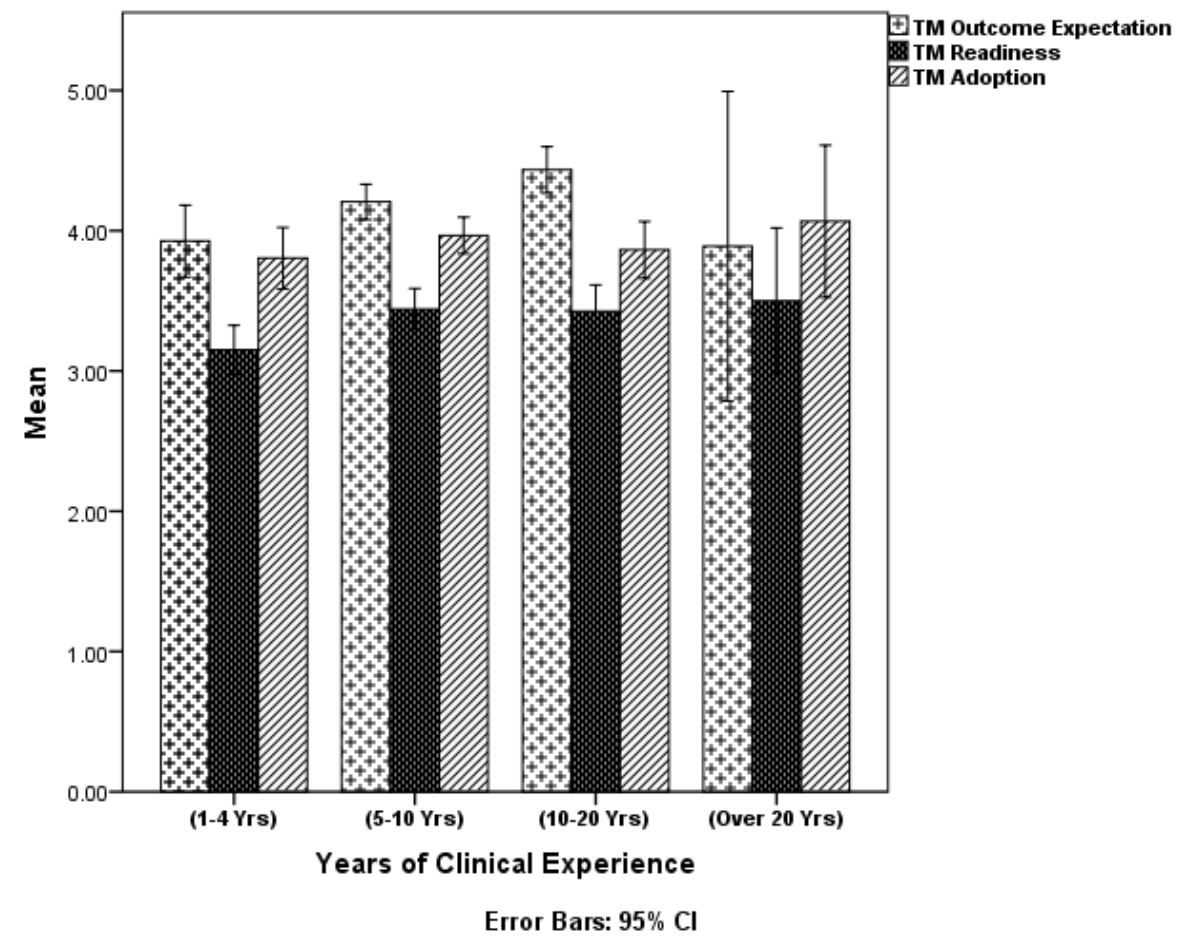

Figure 4. Years of experience (1-4 yrs, 5-10 yrs, 10-20 yrs and over 20 yrs) and telemedicine readiness. $\mathrm{P}=0.044$ 


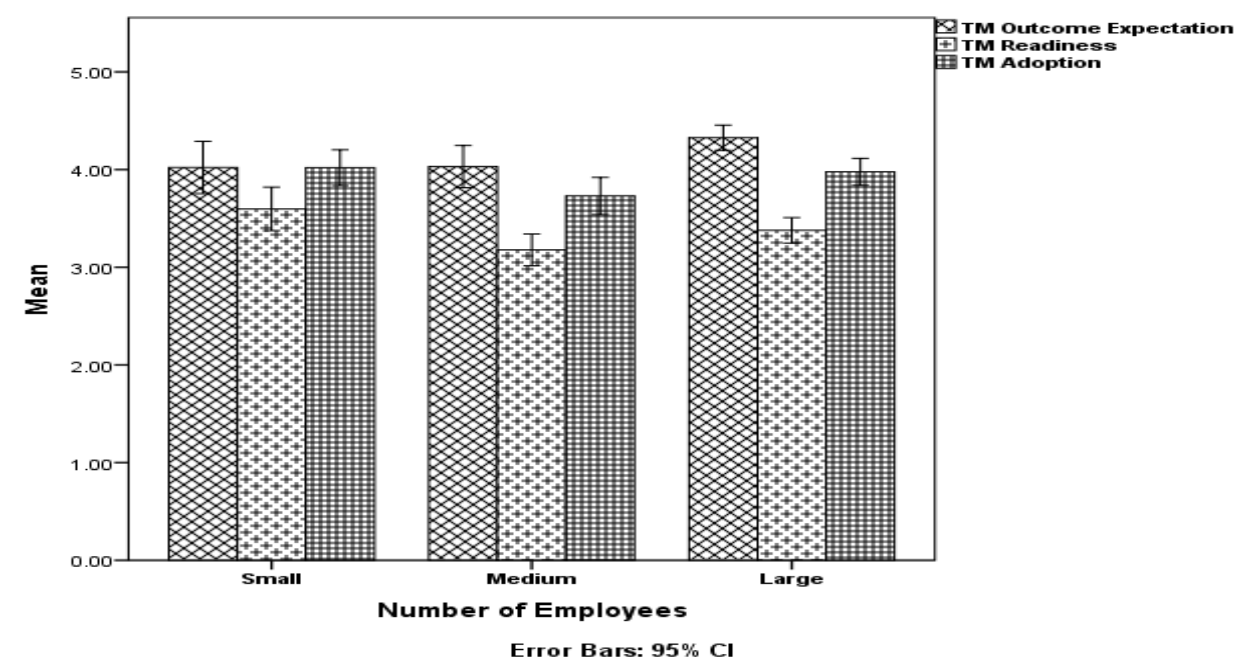

Figure 5. Organisation size ( small $<50$, medium $\geq 51 \leq 250$, large $>500$ ) and telemedicine readiness. $\mathrm{P}=0.006$

TABLE I. RELIABILITY AND CORRELATION TESTS

\begin{tabular}{|c|c|c|c|c|c|c|c|}
\hline \multirow{3}{*}{$\begin{array}{l}\text { Variables } \\
(\mathrm{n}=161)\end{array}$} & \multirow{3}{*}{$\begin{array}{l}\text { Reliability Test } \\
\text { Cronbach's Alpha }\end{array}$} & \multicolumn{6}{|c|}{ Correlations Test } \\
\hline & & \multicolumn{2}{|c|}{ TM Outcome Expectations } & \multicolumn{2}{|l|}{ TM Readiness } & \multicolumn{2}{|l|}{ TM Adoption } \\
\hline & & Pearson's & Sig. (2-tailed) & Pearson's & Sig. (2-tailed) & Pearson's & Sig. (2-tailed) \\
\hline $\begin{array}{l}\text { Service User } \\
\text { Acceptance }\end{array}$ & 0.704 & $.543^{* *}$ & 0.001 & $.330^{* *}$ & 0.001 & $.512^{* *}$ & 0.001 \\
\hline TM Access & 0.781 & $.252^{* *}$ & 0.001 & $.294^{* *}$ & 0.001 & $.264^{* *}$ & 0.001 \\
\hline $\begin{array}{l}\text { HP User } \\
\text { Acceptance }\end{array}$ & 0.877 & $.534^{* *}$ & 0.001 & $.321^{* *}$ & 0.001 & $.654^{* *}$ & 0.001 \\
\hline $\begin{array}{l}\text { HP Learning } \\
\text { Capability }\end{array}$ & 0.777 & $.201^{*}$ & 0.01 & $.280^{* *}$ & 0.001 & $.220^{* *}$ & 0.005 \\
\hline $\begin{array}{l}\text { HP operation } \\
\text { Capability }\end{array}$ & 0.825 & $.444^{* *}$ & 0.001 & $.277^{* *}$ & 0.001 & $.442^{* *}$ & 0.001 \\
\hline IT Infrastructure & 0.866 & $.216^{* *}$ & 0.006 & $.440^{* *}$ & 0.001 & 0.145 & 0.066 \\
\hline $\begin{array}{l}\text { HP HR } \\
\text { Capability }\end{array}$ & 0.884 & $.614^{* *}$ & 0.001 & $.535^{* *}$ & 0.001 & $.521^{* *}$ & 0.001 \\
\hline
\end{tabular}

